\title{
MSTd neurons encode nonlinear combinations of retinal and extra-retinal signals
}

Lukas Brostek $^{1,2}$, Thomas Eggert', Seiji Ono ${ }^{5}$, Michael J. Mustari ${ }^{5}$, Ulrich Büttner ${ }^{2,4}$, Stefan Glasauer ${ }^{1,2,4}$

munich

Bernstein Center for Computational Neuroscience, ${ }^{2}$ Clinical Neurosciences, ${ }^{3}$ Department of Neurology, ${ }^{4}$ Integrated Center for Research and
${ }^{5}$ Washington National Primate Research Center, University of Washington, Seattle, WA, USA

\section{Introduction}

Neuronal activity in the dorsal Medial Superior Temporal area (MSTd) is assumed to depend on both retinal and extra-retinal input. Most of the neurons show activity when presented with a moving large-field visual stimulus (Newsome et al. 1988). This activity is modulated by extra-retinal variables as eye velocity and position (Ben Hamed et al. 2003). However, the interaction between the retinal and extra-retinal input is not yet fully understood. Here we present novel insights regarding Ghe tuning of MSTd neurons for different explanatory variables using an information-theoretic approach

\section{$\sum$}

Information-theoretic approach for determining tuning functions

Weuronal tuning functions can be expressed by the conditional probability $p_{S I V}(S \mid)$ Oof observing a spike $S$ given some explanatory variable $V$. Using Bayes' theorem, - this can be stated as

א్ల

where $p_{V}(v)$ is the probability of observing the explanatory variable and $p_{V, S}(v, s)$ the joint probability of observing variable and spike.

5

For proper estimation of the tuning function it is crucial to take into account the this latency in such a way, that the mutual information I $(V$; $S)$ is maximized.

㐌

\begin{tabular}{cc}
$\infty$ & $I(V ; S)=H(S)-H(S \mid V)$ \\
\hdashline 0 & $H(S \mid V)=-\sum_{v} p_{V}(v) \sum_{s} p_{S \mid V}(s \mid v) \log p_{S \mid V}(s \mid v)$
\end{tabular}

-Randomly moving large-field visual stimulus

INe analyzed the dependence of MSTd neuronal activity in Ononkeys on various retinal and extra-retinal signals during Orith flat vo of a randomly moving large-field visual stimulus Qwith flat velocity spectrum (white noise). The monkey's task Thimus or fixed at a central position. All variables refer to the 要

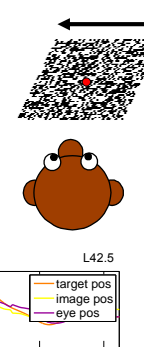

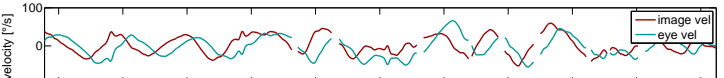

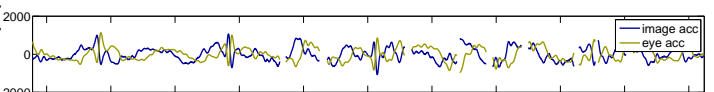

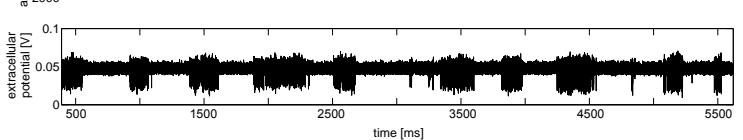

\section{Dependence on a single explanatory variable}

Across a population of 31 MSTd neurons neuronal activity depended on various retinal and extra-retinal signals during the presentation of a randomly moving largefield stimulus. The figure below shows the proportion of mutual information for each explanatory variable and neuron.

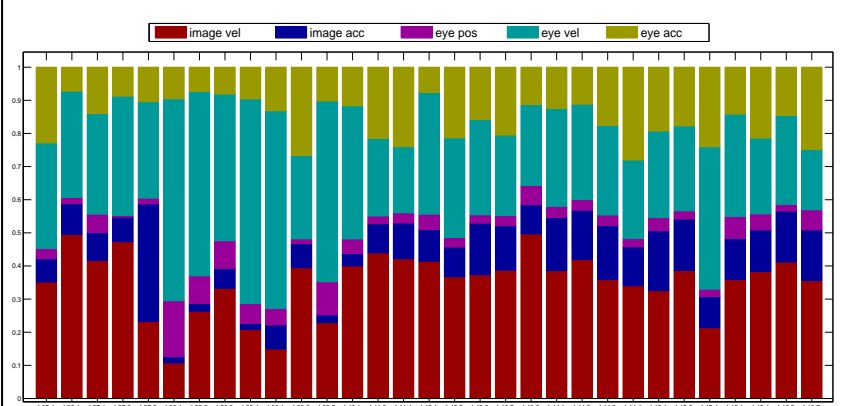

Estimated neuronal latencies of image velocity, eye velocity and eye acceleration were quite consistent across the population. For image acceleration and eye position the
latencies of in atencies of image and eye velocity.

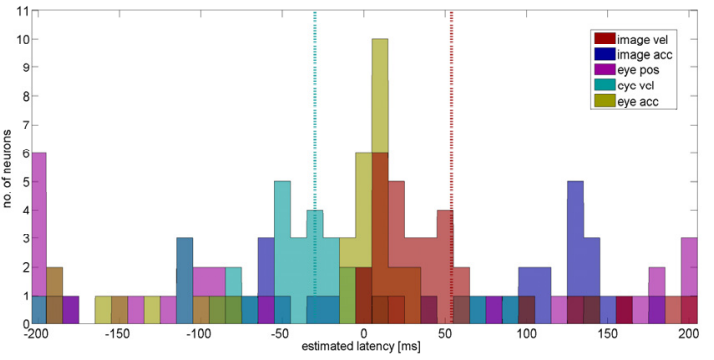

Conclusions

- Spiking activity in MSTd neurons depends nonlinearly on various inputs. - For most neurons the firing rate increases with retinal image velocity and eye velocity. However, many neurons prefer other signals, as eye and image acceleration.

- These findings support the hypothesis that MSTd uses a basis function representation for encoding nonlinear combinations of retinal and extraretinal signals as suggested by Ben Hamed et al. (2003).

Acknowledgements:
Study supported by BMBF (BCCN Munich 01GQ0040, IFB 01 EO 0901), NEI EY06069, RR00165

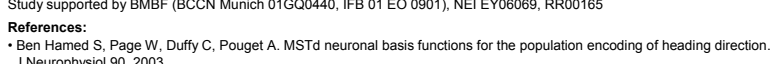

Dependence on combinations of two explanatory variables

Here we analyzed the dependence of spiking activity on a combination of explanatory variables. For each neuron those two variables were chosen that had the highest mutual information in the one-dimensional analysis. Each figure shows the expected firing rate $[\mathrm{Hz}]$ in dependence of these variables. Estimated neurona latencies are noted at the axis labels.
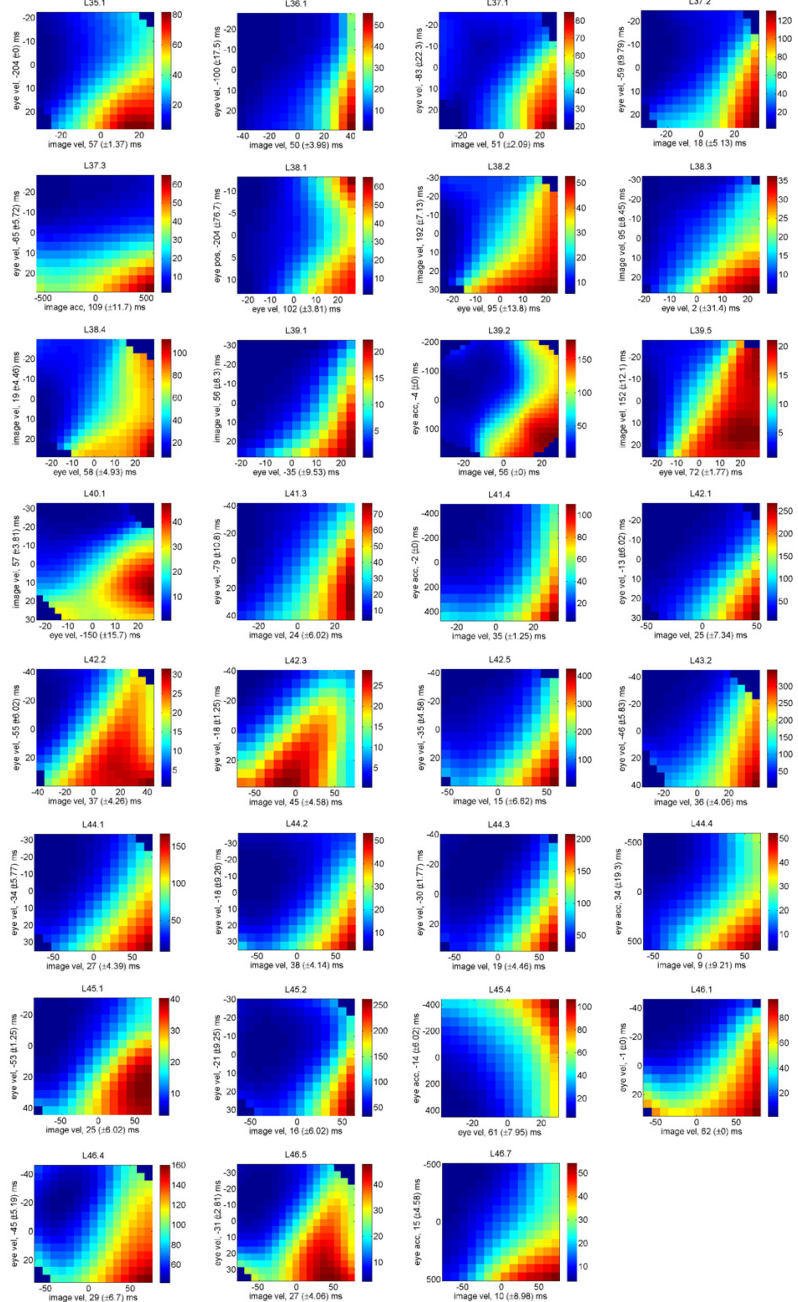

Contact: Lukas.Brostek@lrz.uni-muenchen de 